\title{
Traveling echo waves in an array of excitable elements with time-delayed coupling
}

\author{
Oriol Vallès-Codina, ${ }^{1,2}$ Ronny Möbius, ${ }^{2}$ Sten Rüdiger, ${ }^{2}$ and Lutz Schimansky-Geier ${ }^{2}$ \\ ${ }^{1}$ IBE-Institut de Biologia Evolutiva (UPF/CSIC), Barcelona, Spain \\ ${ }^{2}$ Institut für Physik, Humboldt-Universität zu Berlin, Germany
}

(Received 3 March 2010; revised manuscript received 16 November 2010; published 23 March 2011)

\begin{abstract}
We study time-delayed coupling in one-dimensional arrays of discrete excitable FitzHugh-Nagumo elements. For small time delays in an excitatory coupling, noise-triggered spikes undergo a slight prolongation. For larger delay times, the coupling to adjacent elements leads to a splitting of spikes and an "echo" effect that produces dampened series of spikes. For very large delay times, a transition to a stationary regime appears, as the spikes are sustained by mutual excitations, resulting in antiphase synchronization of all elements. We characterize several features exhibited by the system, such as speed and duration of pulses as well as bifurcations leading to the stationary regime.
\end{abstract}

DOI: 10.1103/PhysRevE.83.036209

PACS number(s): 05.45.Xt, 02.30.Ks, 87.18.Tt

\section{INTRODUCTION}

Systems consisting of coupled excitable elements have been widely studied as models for many natural phenomena [1,2], such as the communication between neurons in the brain. The FitzHugh-Nagumo (FHN) set of equations, as a two-dimensional simplification of the four-variable HodgkinHuxley model, has been proven to be a successful model for the description of the spike generation in the neuronal axon [3,4]. According to the FHN model, a spike may be created locally in an oscillatory or excitatory fashion, depending on a control parameter. In the latter case, one needs noise in order to produce random excitations, which propagate because of coupling of neighboring elements [1]. Coupling is of great importance as it carries the information of the topological distribution of the system. A diffusive coupling, which assumes a mean-field situation, is a useful tool, but more complex kinds of coupling have also been used [5], taking into account the details of connectivity in the system.

In this direction, we took as initial point of research Ref. [6], where we compared the time-delay coupling with the diffusive one in order to show that the former could explain certain properties of spatiotemporal patterns displayed by the intracellular release of calcium. This system provides an important cell-physiological process, where recent experimental and modeling evidence strongly suggests excitable behavior [7]. From this point of view, low-copy number noise in binding and unbinding of molecules governs stimulation of $\mathrm{IP}_{3}$ receptor channels on the membrane of the endoplasmic reticulum (ER). $\mathrm{IP}_{3}$ receptor channels release calcium ions from the ER, partly in response to the binding of $\mathrm{Ca}^{2+}$ to the channel. This constitutes a feedback mechanism [8] that will produce interesting spatiotemporal release patterns. It is important to remark that in many cell types the $\mathrm{IP}_{3} \mathrm{R}$ channels are clustered in groups of channels [9], so that coupling may synchronize channels at two different spatial scales (intracluster or cell-globally), as it is observed experimentally.

In Ref. [6], different behaviors were observed in the dynamics of arrays of excitable elements according to the nature of the coupling, either diffusive or time delayed. In the case of time-delayed coupling, signals from neighboring elements were shown to serve as mutual excitations, resulting in a prolonged signal duration. This suggests an explanation of the two different release modes, local and global, observed in the $\mathrm{Ca}^{2+}$ dynamics, which cannot be found for instantaneous diffusive coupling. In this analysis, an individual cluster was assumed to be one single dynamical element, and its collective dynamics of the channels inside was described by the FitzHugh-Nagumo-type model, as several authors have previously done $[10,11]$. There the FHN model consisted of the usual two differential equations, with one fast activator variable representing, for instance, the calcium concentration, and one slow inhibitor variable that corresponds to the number of inhibited channels.

In this paper, we extend our earlier work by considering genuinely spatial structures by allowing for large domains and translation of excitatory spikes through the domain. We first study the effects of delay coupling on the properties of pulse solutions, such as their propagation speed as well as pulse shape. Propagation of pulses is an important aspect of pattern-forming systems, which is for our system inherited from the standard excitable system. Furthermore, as the delay effects are increased, propagating pulses undergo a transition in their spatial shape. The changes in the pulse shape can be related to the qualitatively different release modes observed in our earlier publication. Finally, we also study the case of large delay times, where a transition to a stationary and spatially coherent regime occurs.

\section{MOTIVATION FOR TIME-DELAY COUPLING AND MODEL EQUATIONS}

Here we give a brief motivation for delay coupling that extends the one for the intracellular calcium system. There, as explained in Ref. [6], delay originates from the finite distance between dynamically active channel clusters, which is about ten times larger than the actual size of the cluster. Between clusters one can assume absence of channels and thus a purely passive behavior of the medium. The delay time is then the diffusive time that calcium, released from one cluster, needs to arrive at the neighbor and to excite it.

Coupling to neighbors with delay has frequently been studied in models for collective neural activity. In neurons, delay results from different processes, such as synaptic delay or transmission delay. Delay was studied in the frame-work of 


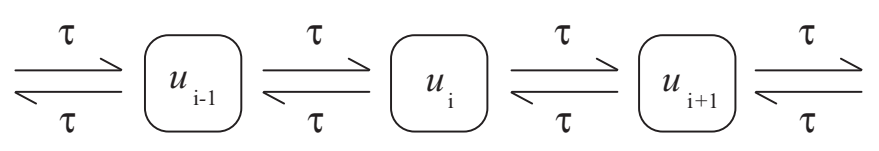

FIG. 1. In our model, excitable elements (boxes) are linked by time-delayed input from adjacent elements to form a one-dimensional array. A delay time $\tau$ arises in neuronal system from the finite propagation speed of signals through connecting axons.

continuous neural fields, where delay results in the interaction function in a distance-dependent manner $[12,13]$. The effects of delay have also frequently been incorporated into integrateand-fire networks $[14,15]$. However, effects of delay-coupling in networks of excitable elements have been much less studied.

Here we consider a one-dimensional network of FitzHughNagumo elements that are coupled to neighbors by delaydiffusive terms (see Fig. 1). The model consists of $N=100$ FitzHugh-Nagumo elements that are coupled with time delay in the activator variable. As we consider only coupling of immediate neighbors in one dimension and periodic boundary conditions, our system may be thought of as a ring of FHN elements. The set of equations is

$$
\begin{gathered}
\frac{\partial v_{i}}{\partial t}=v_{i}\left(1-v_{i}\right)\left(v_{i}-a\right)-w_{i}-w_{0}+\sigma \xi_{i}(t)+C, \\
\frac{\partial w_{i}}{\partial t}=\epsilon\left(v_{i}-\gamma w_{i}\right) .
\end{gathered}
$$

Here $v_{i}$ and $w_{i}$ denote the activator and inhibitor variable, respectively, of element $i$, where $i=1, \ldots, N$. In view of the motivation given above, $v_{i}$ represents the firing function with self-activating local dynamics, while $w_{i}$ is a further quantity representing inhibiting physiological processes. The terms $\sigma \xi_{i}(t)$ stand for fluctuations acting on the system. We will assume the $\xi_{i}(t)$ to be independent Gaussian white-noise processes with vanishing mean and unit variance, and $\sigma$ scales the intensity of the fluctuations.

$C$ denotes the coupling and is given by

$$
\begin{aligned}
C= & c \operatorname{Max}\left[0, v_{i-1}(t-\tau)-v_{i}(t)\right] \\
& +c \operatorname{Max}\left[0, v_{i+1}(t-\tau)-v_{i}(t)\right] .
\end{aligned}
$$

Differently from the kind of systems that were discussed above, we have added a maximum function in the coupling term. The purpose of the maximum value function is to ensure not to have a negative value for the coupling. It thus acts similarly to a diode, allowing only stimulating or excitatory signals on neighbors. An exclusively excitatory coupling may be expected for neurons, which are frequently assumed to enter an excited state upon passing a voltage threshold by input from connected neurons [16]. Another motivation for excitatory coupling originates in the details of calcium release from clusters of channels. Here the clusters are placed relatively distant from each other so that most of the released calcium (i.e., the activator) diffuses into the passive bulk of the cell. Only a small amount of calcium arrives at adjacent clusters but may suffice to activate them. Thus, the disparity between released calcium quantities and arriving calcium quantities establishes an asymmetric coupling, which is accounted for by the maximum function in Eq. (3). A comparison of our results to behavior in systems with similar couplings but without the maximum function will be given in Sec. III D.

Equation (3) amounts to a nondifferentiable right-hand side of differential equations (1). In the language of dynamical systems theory, the equations are a nonsmooth yet continuous system [17]. In the nonsmooth case, special care has to be taken when studying the dynamics, particularly the stability and bifurcations of fixed points. However, conditions for local bifurcations based on eigenvalues can be generalized. Indeed, we show here that instabilities of fixed points are not relevant for the dynamics we observe in simulations. Instead we analyze bifurcations of periodic orbits, where standard bifurcation theory holds in nonsmooth continuous systems.

In order to provide appropriate initial conditions in the simulations, we introduce the coupling for $t \gg \tau$, while before that time the elements are uncoupled. The small $\epsilon$ parameter enforces that the inhibitor variables $w_{i}$ function in a much slower timescale than the activator variables $v_{i}$. Our choice of the two parameters $a=0.1$ and $w_{0}=-0.1$ puts the system on the edge of excitability (the system is Hopf unstable for $w_{0}<w_{0, c}=-0.105$ if $a=0.1$ ). Under the chosen parameters the system has a stable fixed point at $\left(v_{*}, w_{*}\right)=(0.048811,0.097622)$ that corresponds to the rest state. When noise excites the system, pulling it away from the basin of attraction of the rest state, the system starts to perform an excursion along the nullclines to return there, i.e., it fires a spike. As the stability analysis shows, the fixed point possesses complex eigenvalues with a nonvanishing imaginary part, so the decay will proceed in an oscillatory fashion. The FHN model has a Hopf window in the $w_{0}$ values $(-1.24,-0.105)$, where the fixed point has lost stability toward a stable limit cycle. As we will see, although we put the system in the excitatory regime, $w_{0}=-0.1$, the time-delayed coupling, present in the activator variable in Eq. (1), will produce a rather regular oscillatory behavior.

In Fig. 2(a), we plot in the phase space excursions that a single FHN element performs when excited away from its rest state. The characteristic orbit in the phase space is performed along the two nullclines that arise from setting $\dot{v}$ and $\dot{w}$ to zero in Eqs. (1) and (2), with the vertical movement taking much longer than the horizontal one, due to the different timescales of the variables. Since coupled neighboring elements act in an excitatory way, the spiking rate will be larger, and propagation waves are expected to be observed, sustained by the presence of noise.

\section{RESULTS OF NUMERICAL SIMULATIONS}

Typical trajectories of a single element under conditions of time-delayed coupling are shown in Figs. 2(b)-2(d). While panels (a) and (d) show the relatively simple behavior for the limiting cases of vanishing coupling (representing as well typical trajectories in the absence of delay) and large time delay, we found an interesting transition regime in between [(panels (b) and (c)], which we will now discuss in detail. Later we discuss the regular oscillations we found for large time delays [panel (d)].

Like in many other locally excitable systems, diffusive coupling leads to the existence of uniformly translating solutions [18]. The localized perturbation propagates along 

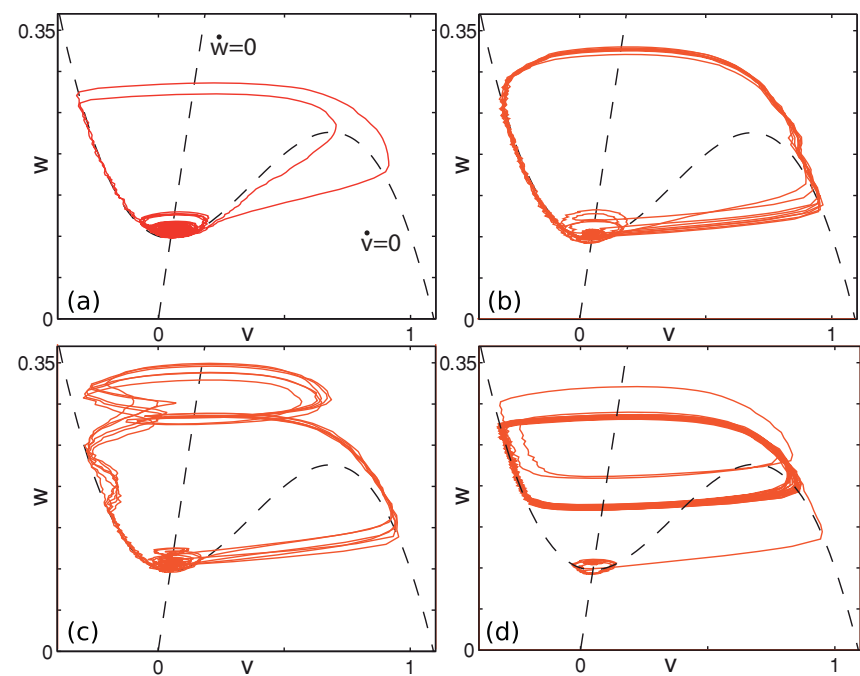

FIG. 2. (Color online) In the phase space of a single FHN element we find, depending on the delay time $\tau$, different qualitative behaviors: Panel (a) corresponds to cycles of an uncoupled element, panel (b) displays the prolonged spike in the solitary wave regime $(\tau=3)$ with a clear vertical expansion of the orbit, panel (c) corresponds to the splitting of the spikes $(\tau=13)$, and panel (d) shows the characteristic orbit of the stationary regime $(\tau=23)$. Here coupling strength was held fixed to $c=0.3$. (Values normally used for the other parameters are $a=0.1, \gamma=0.5, w_{0}=-0.1, \epsilon=0.01$, $\sigma=0.001$, or as indicated if otherwise.)

the spatial coordinate and acquires a fixed shape with a certain spatial extension. We first studied our system by analyzing the dependence on coupling strength and delay time of pulse duration and speed of propagating pulses. We defined the pulse duration $T$ as the time that the fast variable spends over the half of the maximum value $v_{\max }$ in the deterministic spike, i.e., the half-maximum pulse duration. In the absence of coupling ( $c=0$ ), the pulse duration is $T_{0} \approx 27.356$.

We first focus on the dependence of the propagation speed $s$ on the coupling strength $c$ and delay time $\tau$ [Fig. 3(a)]. For comparison with the standard scaling of wave speed with $c$ we will analyze our data double logarithmically, as shown in Fig. 3(b). Accordingly we determine the exponent $\beta$ relating speed and coupling strength by

$$
s \propto c^{\beta(\tau)},
$$

where $\beta(\tau)$ depends on the delay time $\tau$. Note that a power law with exponent $\beta=0.5$ is normally present in the case of purely diffusive coupling. From Fig. 3(b), $\beta(\tau)$ can be obtained as the linear slope for each value or $\tau$. The dependence of $\beta$ on $\tau$ is shown in Fig. 3(c). Here $\beta$ increases for reduced delay and acquires a value of $\approx 0.719$ for vanishing delay time. In other words, the standard diffusive exponent 0.5 is not recovered for vanishing delay time $\tau$, demonstrating a strong effect of the Max function for small $\tau$.

Next we study the dependence of pulse duration $T$ on the parameters $c$ and $\tau$. An unexpected feature that the system displayed was the broadening of pulses with increasing $\tau$ and the existence of a maximum of duration at $\tau \approx 9$ [see Fig. 4(a)]. The increase in pulse duration is larger with increasing coupling constant. However, for larger $\tau>9$ there
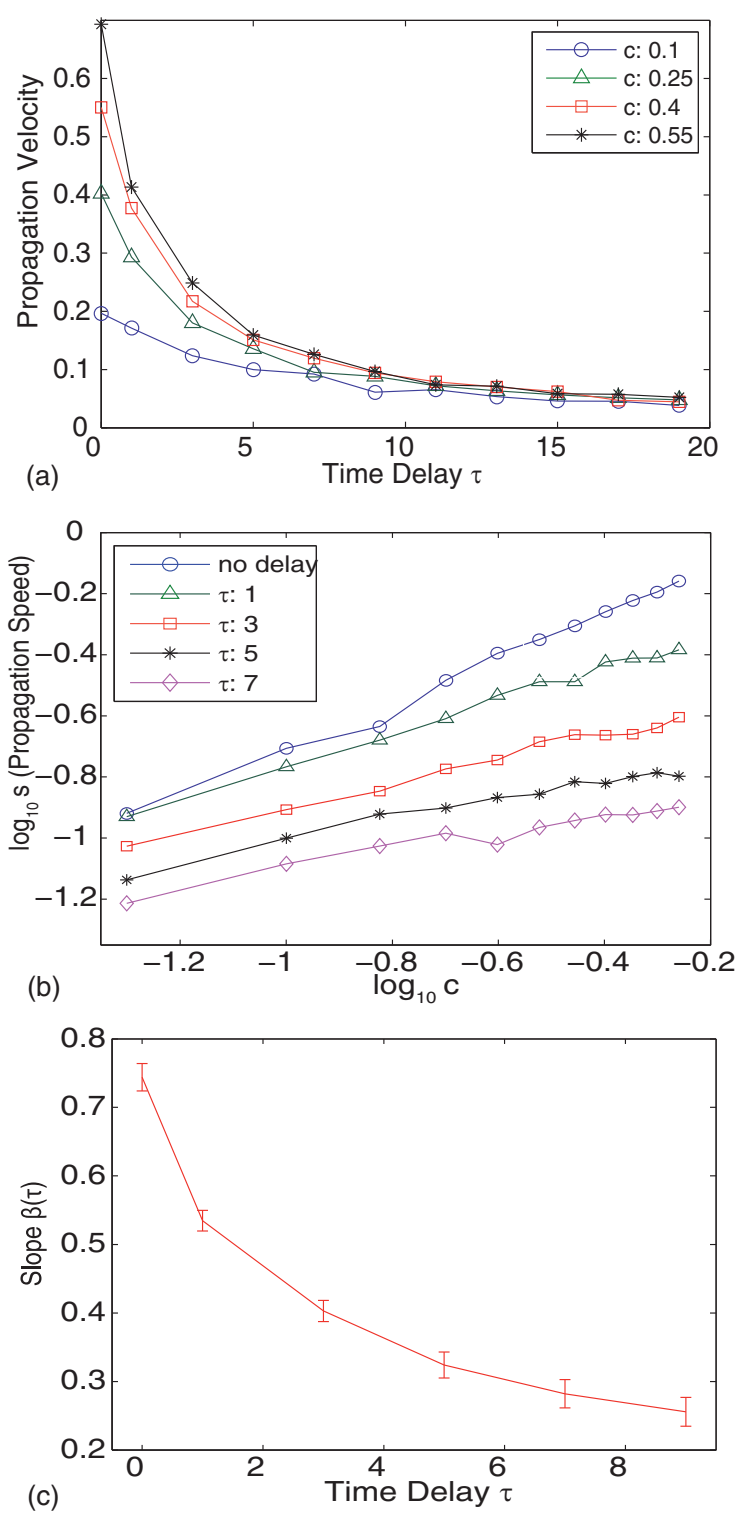

FIG. 3. (Color online) (a) Propagation speed of solitary pulses plotted versus delay time $\tau$. (b) The propagation speed shows a powerlaw behavior with exponent $\beta(\tau)$ when plotted versus the coupling strength $(\sigma=0.005)$. (c) The dependence of exponent $\beta$ on $\tau$.

is a decrease of $T$, and, for even larger $\tau>20$, there is also a reversal in the coupling strength dependence. This complex behavior, as detailed below, is caused by a transition occurring between two very distinct regimes, the first occurring at small delay times (for $\tau<9$ ) and featuring only solitary propagating waves that arise through excitation by noise, and the second one happening for large delay times (for $\tau>20$ ), being a stationary situation where all elements of the system oscillate in an antisynchronous fashion (see below). Between the two regimes, the system experienced a transition sharing some properties with both.

\section{A. Solitary Wave Regime}

In the solitary wave regime (i.e., $\tau<9$ ) pulse duration increases linearly with delay time and coupling strength 


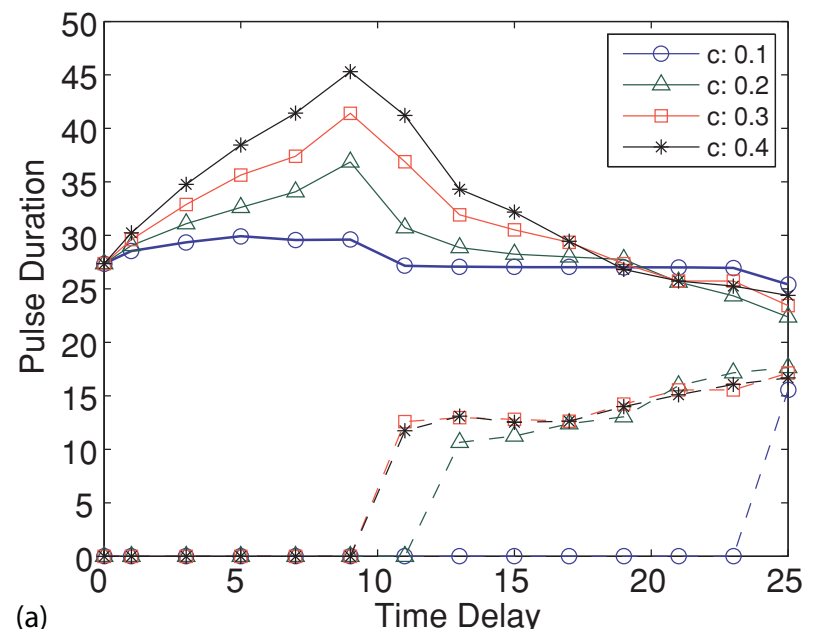

(a)

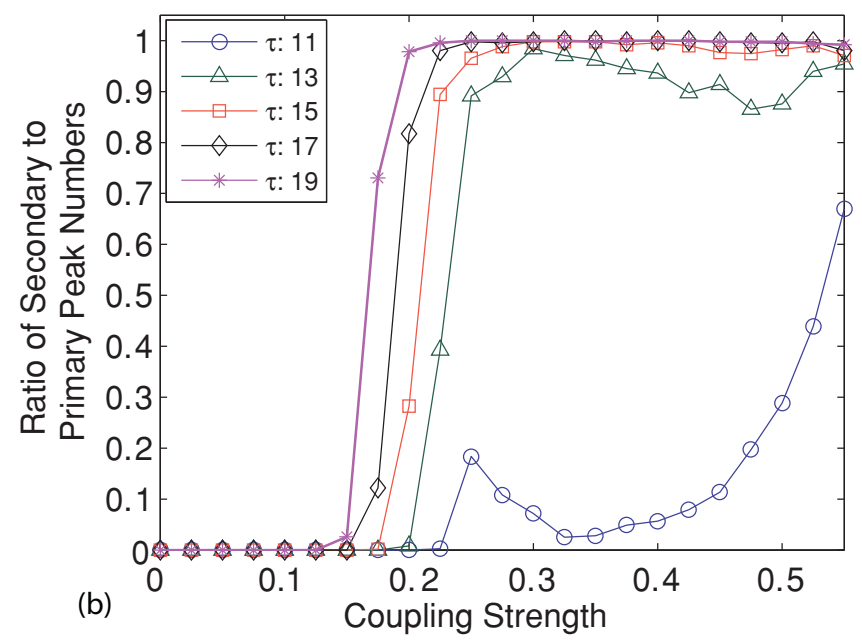

FIG. 4. (Color online) (a) Pulse duration versus delay time. Solid and dashed curves show data for primary and secondary pulses, respectively. (b) Ratio of secondary to primary peaks for each simulation. Secondary pulses appear for large coupling strength, where the critical $c$ is shifted toward lower values for increasing delay time. For all simulation we here used $\sigma=0.01$.

[Fig. 4(a)]. Furthermore, if we plot the pulse duration $T$ normalized by $\tau$ versus coupling strength (i.e., $T / \tau$ versus $c$ ), the straight lines become parallel (data not shown), so that the analytic expression must be of the type

$$
T=T_{0}+\alpha c \tau
$$

where $\alpha$ is positive and independent of $\tau$ and $c$. Here $T_{0}$ is the pulse width without delay as defined above. By linear fitting we find $\alpha=(5.38 \pm 0.19)$ with a deviation of $3.5 \%$.

The widening effect of an increasing time delay is understood in an intuitive way, as excited neighbors affect later (in a delayed way) the spike of the element, so that the pulse is sustained longer. However, if we continue increasing the time delay, we reach a point where the spike cannot grow any wider and splits, initiating the transition to the stationary regime.

\section{B. Transition regime: Echo waves}

In the transition regime (delay times $9<\tau<20$ ), the delay is too large to sustain a long spike, affecting the neighboring elements by making them spike again just after a normal excitation. The transition is illustrated in Fig. 5: While for small delay $\tau=3$ an individual element exhibits single spikes of equal amplitude [panels (a) and (e)], for larger $\tau=13$ a short series of dampened spikes appear [panels (b) and (f)]. This corresponds to the generation of a second-time scale, namely, the slow decay of spiking activity studied in Ref. [6].

We can distinguish between the main pulse, which we call primary, and the shorter or secondary ones caused by the delay, as observed in Figs. 5(b) and 5(c) and corresponding to solid and dashed graphs, respectively, in Fig. 4(a). The delay-induced effect resembles an echo and strengthens with increasing delay time, as new spikes appear after the secondary peaks due to the feedback. This echo effect maintains smallamplitude spikes until they vanish or are overtaken by the next solitary wave. In Fig. 2(c), it is possible to see that the main pulse corresponds to the wider and lower orbit [which appears to resemble the original un-coupled orbit of Fig. 2(a)], while the secondary pulse is the upper and shorter.

Furthermore, in this regime the pulse durations show a dependence on the coupling strength as well. From Fig. 4(b), the transition to secondary pulses can be seen to occur at specific values $c_{0}(\tau)$. Specifically, the splitting of spikes begins with lower coupling strengths $c_{0}(\tau)$ as the delay time increases. This feature can be related to the bifurcation behavior investigated in Sec. IV.

In this fashion, increasing the time delay increases the length of the echo until we reach $\tau \approx 20$, when the transition ends as the last secondary spikes hit the first next main spike. Here the stationary regime appears.

\section{Stationary Regime}

For large delay times $(\tau>20)$, the secondary spikes generated by the echo effect live long enough to contact the next propagating wave. The outcome is an antiphase synchronization of all elements, which can be likened to $\pi$-mode oscillations [Fig. 5(h)]. For increasing delay time and after transients, this phenomenon extends to the complete chain of elements.

As shown in Fig. 2(d), the orbit in the phase space has evolved to a very specific cycle along the nullclines, which is shorter and with smaller amplitude [see also Fig. 5(d)] than the original uncoupled one. This situation corresponds clearly to a stationary regime, where we recover only one specific time duration of spikes.

As we can see from Figs. 2(d) and 5(d), the orbit in the phase space corresponds to an oscillatory state of every element of the system. It carries a specific duration for the $v$ variable, which corresponds to the time that the system spends on the right branch of the trajectory [i.e., the vertical width of the orbit; see Fig. 2(d)], which is clearly shorter than in the solitary wave regime. Thus, as the coupling establishes the stationary regime, an increase of the coupling strength implies a decrease in the pulse duration [see Fig. 6)]. Thus, the widening effect 

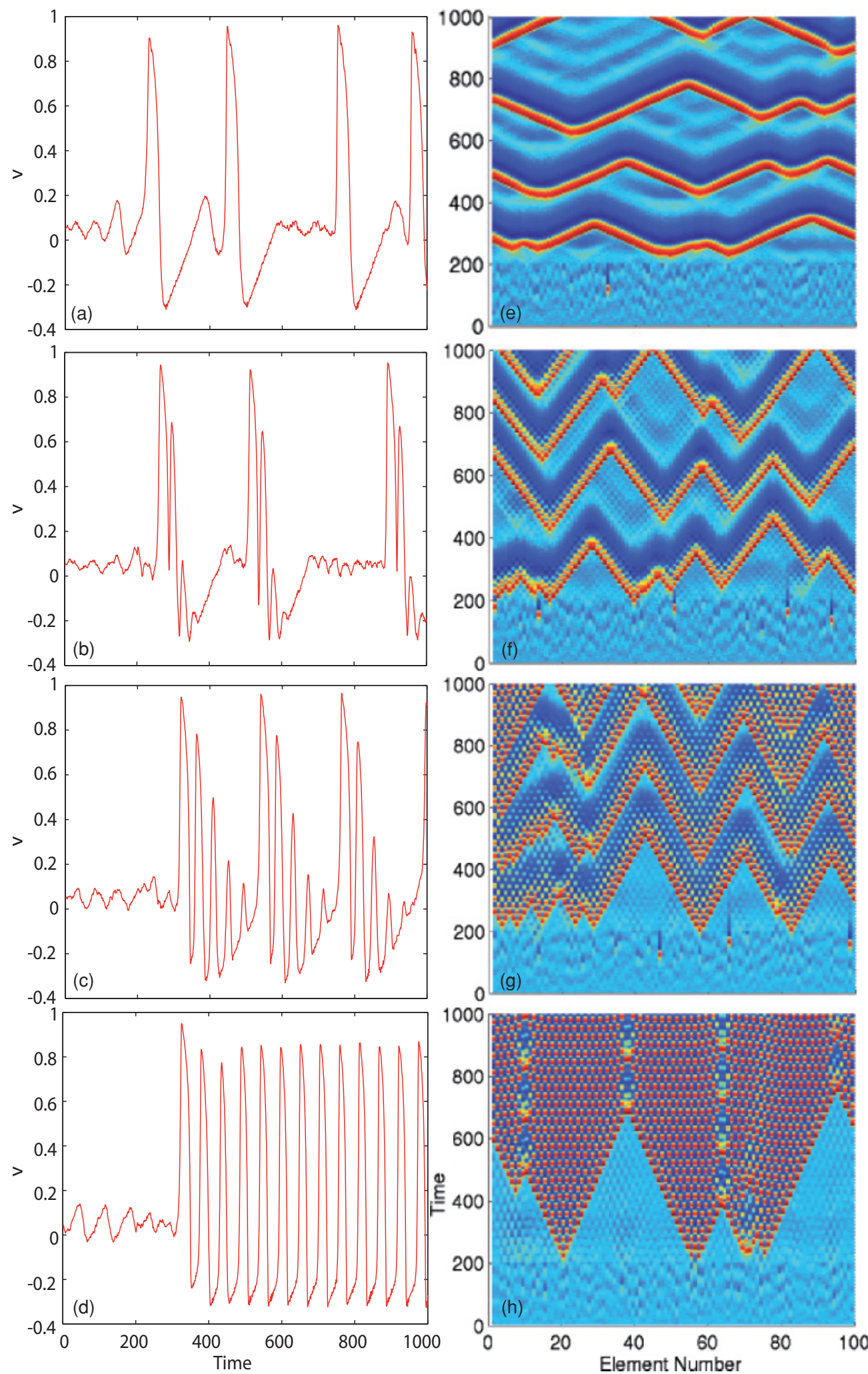

FIG. 5. (Color online) Change in the behavior of a single element $(\mathrm{a}-\mathrm{d})$ and of the entire system $(\mathrm{e}-\mathrm{h})$ for increasing delay times $[\tau=3(\mathrm{a}$, e), $13(\mathrm{~b}, \mathrm{f}), 19(\mathrm{c}, \mathrm{g})$, or $25(\mathrm{~d}, \mathrm{~h}), c=0.3, \sigma=0.01]$.

due to the echo effect is completed by a synchronization regime, where echoes of equal amplitude reduce the refractory state.
The homogeneous nature of the antiphase oscillation allows, as was done in Refs. [19-21], the phenomenon to be modeled as the alternate firing of just two FHN elements. This 


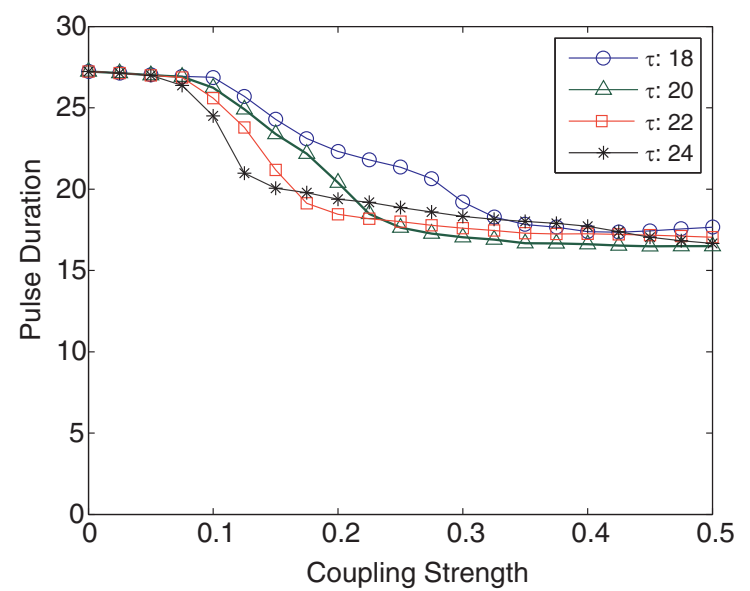

FIG. 6. (Color online) For large time delays, the pulse duration decreases with an increase of the coupling, so that it bears a different dependence than in the other regimes.

idea will be taken up in Sec. IV by the study of bifurcations in a two-element system.

\section{Other kinds of time-delayed coupling}

After using (3) to define the coupling in our simulations, we turn our attention as well to other kinds of time-delayed coupling,

$$
\begin{gathered}
C=c v_{i-1}(t-\tau)-2 c v_{i}(t)+c v_{i+1}(t-\tau), \\
C=c v_{i-1}(t-\tau)+c v_{i+1}(t-\tau),
\end{gathered}
$$

where we omit the Max function in (5), so that the coupling now resembles a diffusive coupling with a time-delayed effect. For (6), the $2 v_{i}$ term vanishes, in effect removing the local part of the coupling function.

In the case of coupling (5), the combined diffusive timedelayed kind, we allow its values to be negative, so that there is a competition between noise and the coupling (we set the amplitude of noise to $\sigma=0.03$ from here on). While the former factor increases the probability for an element to be excited and sustains the propagation of waves, the latter will often be negative due to the $2 c v_{i}$ term, which generally stabilizes the fixed point.

Interestingly, again a stationary regime with $\pi$-mode oscillations arises, but with the significant difference that it does not appear after a propagation wave regime as before. In contrast, it emerges eventually as an amplification of the noise in the rest state and as soon as $\tau=5$ or larger. As well, we see that the coupling strength plays here a key role: For small values of $c$ (i.e., $c<0.1$ ), the noise prevails and we have a scenario with single elements spiking with little correlation. By increasing the coupling strength, the system enters a regime where the coupling dominates in a stabilizing way and there is no excitation in any element. Then, above a certain critical value of the coupling strength $c_{c}(\tau)$, the system generates a stationary regime, consisting of antisynchronous oscillations similar to the one in the previous section, but that emerge by a more fluctuating propagation through space (Fig. 7).

It is worth comparing this with Ref. [21], as those authors state that noise-induced and delay-induced oscillations in
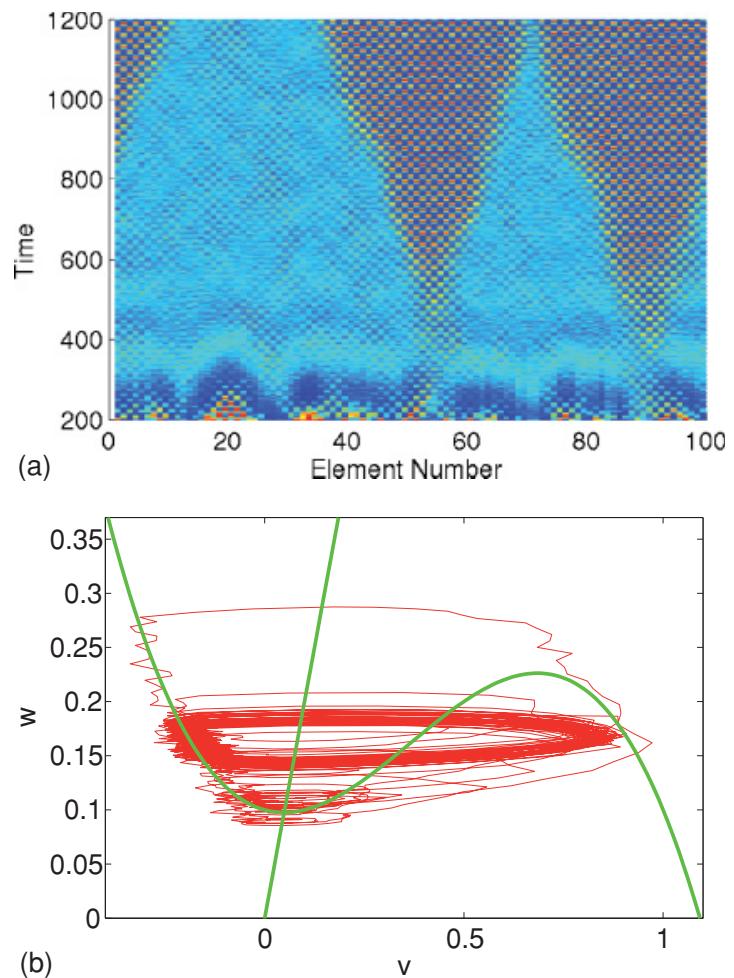

FIG. 7. (Color online) Initiation of the stationary regime for the coupling (5) (without the Max function).

excitable systems are inherently different. In our simulation, we observed how noise eventually creates the oscillations from the (stable) rest state to reach the usual spiking mode. Consequently, noise and time delay are at interplay, with noise producing the gradual emergence of the stationary oscillations and time delay keeping the oscillations sustained (as in Sec. III C or Ref. [22]).

In the other case, the coupling (6) has no negative term, so we expect no competition between noise and coupling this time. In fact, we obtain only one regime for all time delays, which features very correlated and long oscillations of all elements of the system. The coupling does not only help to synchronize the system, but also allows the stronger mutual interactions to sustain longer spikes. In this last aspect, the coupling without the $v_{i}$ term features a similar behavior as our original coupling.

\section{LINEAR STABILITY AND BIFURCATION ANALYSIS}

In the following we will describe in a detailed way the bifurcation scenario for stationary antiphase oscillations. For simplicity we begin with the linear stability of the fixed-point solution in the case without Max function. The equations we will now study are

$$
\begin{gathered}
\frac{\partial v_{j}}{\partial t}=f\left(v_{j}\right)-w_{j}+c\left[v_{j-1}(t-\tau)-2 v_{j}(t)+v_{j+1}(t-\tau)\right] \\
\frac{\partial w_{j}}{\partial t}=\epsilon\left(v_{j}-\gamma w_{j}\right)
\end{gathered}
$$

where $f(v)=v(1-v)(v-a)-w_{0}$. Linearizing around a constant solution $v_{j}^{0}, w_{j}^{0}=$ const. in terms of perturbations 
$\delta v, \delta w$ and assuming wavelike perturbations $\delta v_{j}=\hat{v} \exp (\lambda t+$ $i q j), \delta w_{j}=\hat{w} \exp (\lambda t+i q j)$, we obtain

$$
\lambda \hat{v}=\left\{f^{\prime}+c[\exp (-\lambda \tau-i q)+\exp (-\lambda \tau+i q)-2]\right\} \hat{v}-\hat{w}
$$

$$
\lambda \hat{w}=\epsilon(\hat{v}-\gamma \hat{w}),
$$

which we aim to solve for the growth rates $\lambda$. Here $q$ denotes the spatial wavenumber of the perturbation and $f^{\prime}$ denotes the derivative of $f$ with respect to its argument. In the simplest case, $q=0$, which corresponds to spatially homogeneous perturbations, the characteristic equation is

$$
(\lambda+\epsilon \gamma)\left\{\lambda-f^{\prime}+2 c[1-\exp (-\lambda \tau)]\right\}+\epsilon=0 .
$$

Note that if $c=0$ (no coupling) or $\tau=0$ (standard diffusive coupling), the resulting condition for bifurcation is $f^{\prime}=\epsilon \gamma$ with stability for $f^{\prime}<\epsilon \gamma$. If, however, $c>0$ and $\tau>0$, the term $2 c[1-\exp (-\lambda \tau)]$ does not vanish and can in general destabilize the fixed point. Similarly, for an antisynchronous perturbation $(q=\pi)$ we obtain the characteristic equation

$$
(\lambda+\epsilon \gamma)\left\{\lambda-f^{\prime}+2 c[1+\exp (-\lambda \tau)]\right\}+\epsilon=0 .
$$

For small $\tau$ one can expand the last two equations to obtain $(\lambda+\epsilon \gamma)\left\{\lambda-f^{\prime}+2 c[1 \pm(1-\tau \lambda)]\right\}+\epsilon=0$, but general solutions have to be found numerically. For our set of parameters we did not find evidence of coupling-induced instability. This conclusion was also obtained in Ref. [20] for a similarly structured dynamical system.

Considering now the original system with Max function, we realize that it is located in between the uncoupled one and the diffusively coupled one. According to the general theory for nonsmooth dynamical systems, local bifurcations may be qualitatively different from those of smooth systems. However, they are equally indicated by changes in eigenvalues of the linearized equations, in the sense that a significant eigenvalue crosses the imaginary axis [17]. The fact that for the diffusively coupled system no coupling-induced instability occurs suggests the lack of instability for the system with Max function. Therefore, and from what will be shown below by branch continuation, we conclude that the periodic solution is not related by instability to the fixed point.

To elucidate the origin of periodic, antiphase solutions, we next study their branching behavior. We here restrict ourselves to the investigation of a symmetry-adapted subspace. Because of the spatial periodicity of our antiphase solution, it can be considered as a stable, temporal oscillation of two coupled elements. The equations we will now study are

$$
\begin{aligned}
\frac{\partial v_{1}}{\partial t}= & v_{1}\left(1-v_{1}\right)\left(v_{1}-a\right)-w_{1}-w_{0} \\
+ & 2 c \operatorname{Max}\left[0, v_{2}(t-\tau)-v_{1}(t)\right], \\
& \frac{\partial w_{1}}{\partial t}=\epsilon\left(v_{1}-\gamma w_{1}\right), \\
\frac{\partial v_{2}}{\partial t}= & v_{2}\left(1-v_{2}\right)\left(v_{2}-a\right)-w_{2}-w_{0} \\
+ & 2 c \operatorname{Max}\left[0, v_{1}(t-\tau)-v_{2}(t)\right], \\
& \frac{\partial w_{2}}{\partial t}=\epsilon\left(v_{2}-\gamma w_{2}\right) .
\end{aligned}
$$

We use the branch-continuation software DDE-BIFTOOL [23] to determine the range of existence and stability of the periodic antiphase orbit.

The applicability of straightforward branch-following methods to nonsmooth, continuous systems is facilitated by the following argument. Bifurcations of periodic solutions are generally analyzed in terms of Poincaré sections, where repeated intersections of periodic solutions with appropriately chosen spaces are determined [17]. Integration of differential equations of nonsmooth but continuous nature therefore results in Poincaré maps with a continuous derivative, which generally allows application of standard bifurcation theory. Further, numerical calculation of periodic branches and their stability (Floquet multipliers) is implemented by a collocation method in BIFTOOL, which does not require derivatives of right-hand sides. We have checked exemplary periodic solutions and the points where they become unstable by detailed comparison with direct numerical simulations and found accurate correspondence.

The system of Eqs. (13)-(16) allows for the standard fixedpoint solution $v_{1}=v_{2}=v_{*}, w_{1}=w_{2}=w_{*}$. As suggested by our numerical simulations in Sec. III, we found a family of stabilized nontrivial solutions with the characteristic feature of antiphase oscillation. Figure 8 shows the amplitude of those periodic solutions for various values of the parameters $\tau$ and $c$. A branch of periodic orbits (solid lines) is connected with a branch of periodic orbits of smaller amplitude (dashed lines). Both branches collide in saddle-node bifurcation at small $\tau$, such that a threshold in delay times $\tau$ exists, below which no periodic orbit occurs. For small $c$ we also found further saddle-node bifurcation of the periodic orbits. As shown in Fig. 8, islands of periodic solutions exist at small coupling strength. If $c$ is increased, the island at $\tau \approx 40$ is connected to the solutions at $\tau \approx 70$. Similarly, further numerical analysis showed that at larger $\tau$ additional islands exists (if $c$ is small).

The two branches produced in a saddle-node bifurcation generally have different stability properties. We found that the lower branch is always unstable, while the upper branch looses stability by a further bifurcation. Stability of periodic orbits is determined by Floquet multipliers. We observed

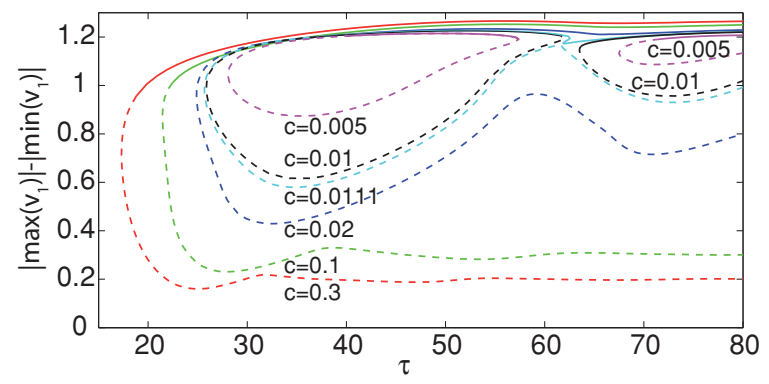

FIG. 8. (Color online) Amplitudes of periodic orbits for Eqs. (13)-(16) (solid lines = stable, dashed lines = unstable) for different values of the coupling constant $c$. The two solution branches of different amplitude collide in saddle-node bifurcations. Further saddle-node bifurcation create islands of periodic solution for small $c$. A pitchfork bifurcation of periodic orbits is responsible for the change of stability along the upper branch. 


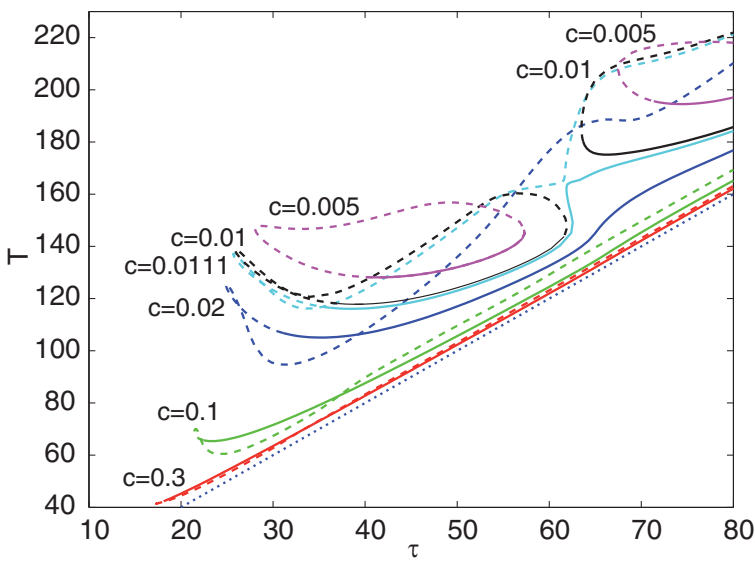

FIG. 9. (Color online) Periods of the same periodic orbits as shown in Fig. 8. Strong deviations from a period $T=2 \tau$ (dotted line) occur if the islands shown in Fig. 8 exist.

that at the stability changing point one multiplier crosses the unit circle at 1 . Accordingly the periodic orbit loses stability in a pitchfork bifurcation of limit cycles, presumably breaking the antisymmetry between the two elements. We could not find any stable periodic solutions bifurcating at this point and thus conclude that the pitchfork bifurcation is subcritical.

Figure 9 shows the period of the antiphase solutions for the same branches as in Fig. 8. For a resonant situation, where pulses alternate in the two elements [corresponding to the time-asymptotic regime in Figs. 5(d) and 5(h)] one would expect the period to be twice as large as the delay time $\tau$. This is indeed roughly the case for large coupling, $c>0.1$. For small coupling, i.e., if the islands exist, periods can last around five times $\tau$. We checked that this behavior is not related to a symmetry breaking between the two elements: their phase difference is always $\pi$.

These results can be summarized to the bifurcation diagram shown in Fig. 10. Stable periodic solutions exist only above

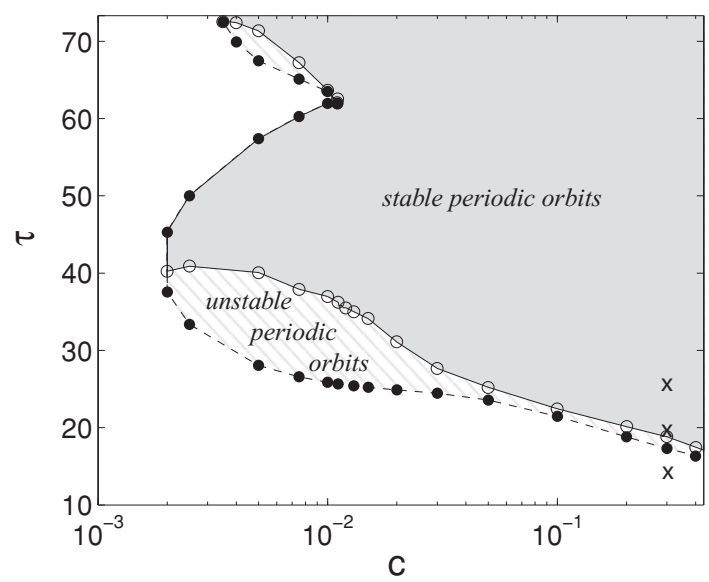

FIG. 10. Location of saddle-node bifurcation (filled circles) and pitchfork bifurcation (open circles) of antiphase oscillations in parameter space. Stable periodic orbits exist to the right and above the solid lines. Crosses show the location of parameters for simulations shown in Figs. 5(f)-5(h) (from bottom to top). and to the right of the solid curve. Particularly, for $c=0.3$ we obtain a minimal $\tau$ for the existence of periodic solutions of around 17, consistent with the onset of stationary antiphase oscillations found in Sec. III. For comparison we have added crosses in Fig. 10 to represent the simulations shown in Figs. 5(f)-5(h) (from bottom to top).

The islands are limited by saddle-node bifurcations (filled circles) and lead to the folds on the left boundary in Fig. 10. Areas between the solid and dashed lines contain unstable periodic orbits, which become stabilized by pitchfork bifurcations (open circles).

In the entire parameter space the fixed point is also stable, leading to bistability of fixed point and periodic solutions. Thus it requires noise or hard excitations to obtain the transition from the fixed point to the periodic orbit at the solid curve. Therefore, a quantitative agreement of bifurcation analysis and the simulation results presented in the first part of the paper is hard to achieve.

We finally note that the pitchfork bifurcation (open circles in Fig. 10) was not found for the case of delay-diffusive coupling without the Max function. The stability region of periodic orbits is therefore substantially different for the two cases with and without Max function.

\section{SUMMARY}

We have described a very uncommon behavior in a timedelay coupled FHN chain, which consists of a transition between a solitary wave regime (small delay times) and a stationary one (large delay times), with clearly different features, specifically for the pulse duration. We determined numerically the scaling behavior connecting the pulse duration and speed with the basic coupling parameters, which are surprisingly accurate. We have also characterized the echo waves, which appear in a regime between the simple pulses and the stationary solutions.

The stationary regime was further studied by using numerical branch continuation. We found a complex behavior of periodic orbits, which are generated in saddle-node bifurcations. Since the original fixed point stays stable, noise plays a dominant role in the transition from trivial to complex patterns. From this point of view, the existence of a dampened series of spikes in the small- $\tau$ regime can be interpreted in analogy to the standard excitability. Noise enforces a perturbation away from the fixed point, with the system being driven to the proximity of the periodic orbits, where it remains for a few cycles, and finally accumulating enough inhibitor to return to the rest state.

\section{ACKNOWLEDGMENTS}

We would like to thank M. Zaks and R. I. Leine for advice. S.R. and L.S.-G. thankfully acknowledge support from the Berlin-Bernstein Center Berlin for Computational Neuroscience. O.V. wishes to thank the Fundación General de la Universidad de Valladolid for the financial support provided within the Faro Global program. 
[1] B. Lindner, J. García-Ojalvo, A. Neiman, and L. SchimanskyGeier, Phys. Rep. 392, 321 (2004).

[2] F. Sagués, J. M. Sancho, and J. García-Ojalvo, Rev. Mod. Phys. 79, 829 (2007).

[3] R. A. FitzHugh, Biophys. J. 1, 445 (1961).

[4] J. Nagumo, S. Arimoto, and S. Yoshitzawa, Proc. IRE 50, 2061 (1962).

[5] E. Alvarez-Lacalle and E. Moses, J. Comput. Neurosci. 26, 475 (2009).

[6] S. Rüdiger and L. Schimansky-Geier, J. Theor. Biol. 259, 96 (2009).

[7] M. Falcke, Adv. Phys. 53, 255 (2004).

[8] S. Zeller, S. Rüdiger, H. Engel, J. Sneyd, G. Warnecke, I. Parker, and M. Falcke, Biophys. J. 97, 992 (2009).

[9] S. Rüdiger, C. Nagaiah, G. Warnecke, and J. W. Shuai, Biophys. J. 99, 3 (2010).

[10] Y.-X. Li and J. Rinzel, J. Theor. Biol. 166, 461 (1994).

[11] L. Meinhold and L. Schimansky-Geier, Phys. Rev. E 66, 050901(R) (2002).
[12] A. Hutt, M. Bestehorn, and T. Wennekers, Netw. Comput. Neural Syst. 14, 351 (2003)

[13] A. Hutt and F. M. Atay, Phys. Rev. E 73, 021906 (2006).

[14] G. Golomb and G. B. Ermentrout, Netw. Comput. Neural Syst. 11, 221 (2000).

[15] H. Haken, Eur. Phys. J. B 18, 545 (2000).

[16] R. Osan and B. Ermentrout, Phys. D 163, 217 (2002).

[17] R. I. Leine and H. Nijmeijer, Dynamics and Bifurcations of Non-Smooth Mechanical Systems (Springer, Berlin, 2004).

[18] J. Hizanidis, A. Balanov, A. Amann, and E. Schöll, Phys. Rev. Lett. 96, 244104 (2006).

[19] N. Buric and D. Todorovic, Phys. Rev. E 67, 066222 (2003).

[20] E. Schöll, G. Hiller, P. Hövel, and M. A. Dahlem, Phil. Trans. R. Soc. A 367, 1079 (2009).

[21] M. A. Dahlem, G. Hiller, A. Panchuk, and E. Schöll, Int. J. Bifur. Chaos 19, 745 (2009).

[22] S. Sailer, D. Hennig, V. Beato, H. Engel, and L. SchimanskyGeier, Phys. Rev. E 73, 056209 (2006).

[23] K. Engelborghs, T. Luzyanina, and D. Roose, ACM Trans. Math. Softw. 28, 1 (2002). 